\title{
Development of printed RFID sensor tags for smart food packaging
}

\author{
Edsger Smits, Jeroen Schram, Matthijs Nagelkerke, Roel Kusters, Gert van Heck, Victor van Acht, \\ Marc Koetse, Jeroen van den Brand, Gerwin Gelinck, Herman Schoo \\ Holst Centre, High Tech Campus 31, 5656 AE Eindhoven, The Netherlands, \\ edsger.smits@tno.nl
}

\begin{abstract}
Sensors integrated into food packages could benefit consumers by ensuring freshness and quality while allowing retail industry to more efficiently manage food stocks and product authenticity. Here we present smart radio-frequency labels with sensors able to measure temperature, humidity and the presence of volatile amine compounds. The labels are made via high quality screen printing and lamination technologies on low cost foils in combination with pick and place technology. As a case study the smart labels are used to quantify the freshness of fish.
\end{abstract}

Key words: radio frequency identification, smart packaging, system in foil, resistive sensors in foil, amine sensor, humidity sensor

\section{Introduction}

All segments of the supply chain can take advantage of wireless (sensor) technology. Retailers in general merchandise, grocery, apparel and other categories are now piloting RFID programs and reporting improved sales from greater stock availability, cost savings and increased responsiveness, especially in receiving and inventory control operations[1]. Current RFID tags are based on chips containing a memory that can be wirelessly read out to uniquely identify products. As these applications continue to prove their value, labels with additional functionality such as timetemperature, pressure, tilt monitoring and chemical sensing are emerging. This requires low-cost sensor development[2]. Monitoring of goods is especially relevant for the transport of perishable goods, such as fruit, vegetables, meats and fish. These products need to be kept under very strict conditions (e.g. temperature, humidity) to arrive fresh at their destination. Faulty handling can result in unsellable products and in potential health risks. Meats and fish are very susceptible food products due to the rapid growth of bacteria in these products. Bacterial growth on meats and fish typically causes the excretion of nitrogen and sulfur containing compound, which is accompanied by strong and foul smell. For example, for fish a marker indicative for deterioration is trimethylamine (TMA) [3]. For practical applications, low concentrations of such nitrous compound, for example in the order of parts per million ( $p p m)$ need to be accurately and reliably measured[4].

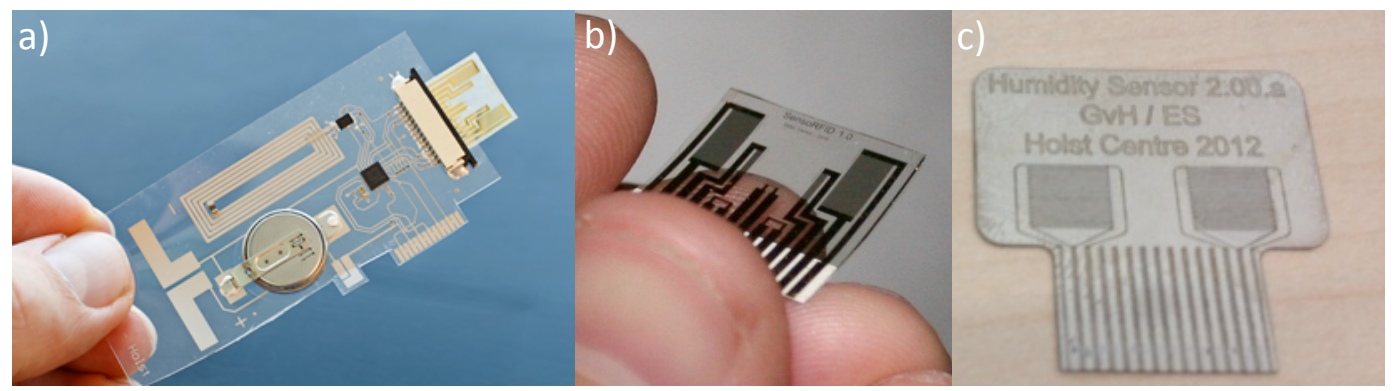

Figure 1:a) Photograph of a 'Smart Label', The complete system includes the printed circuitry, $I C$ 's and a foil containing the sensor elements. b) Photograph of sensors on PEN foil comprising of interdigitated Au electrodes functionalized with sensing polymers. c) Photograph of sensors fabricated by screen printed interdigitated silver electrodes on PET foil and functionalized with sensing polymers. 
In our contribution we discuss the development and fabrication of demonstrators for smart food packaging. A photograph of such a tag is given in figure 1. The goal is to develop Radio Frequency based self-adjusting expiration date labels (Smart Label).

\section{Radio frequency identification sensor tags}

From a system point of view the application contains the following basic building blocks: sensors, logic, radio, antenna and power. We used generic technologies for the low cost assembly and fabrication of systems in foil.

A key technology is high quality screen printing of silver structures serving as the circuit board and the antenna. For usage as circuit board and antenna, the silver lines need to exhibit low resistance and good mechanical stability. Here silver paste cured at low temperature was used to enable fabrication on low costs PEN foils. The limitation in thermal stability of the PEN foil $\left(130^{\circ} \mathrm{C}\right)$ complicates well established processes for making electronic products. For the assembly of and interconnection of passive components and integrated circuits conductive adhesives are needed. Anisotropic conductive adhesives (ACA) were deposited by stencil printing to obtain interconnections. The adhesive exhibits enough electrical conduction as well as mechanical stability for the IC's and passive components of the smart labels[5].

For the logic, commercially available cost effective MSP430G microprocessors from Texas Instruments were used. The microprocessor has the advantage of low power consumption and can be switch to a sleep mode in between the measurement. The microprocessor's multiple 10 bit ADC voltage inputs enable accurate readout of multiple resistive elements and temperature can be measured with an integrated thermometer. To convert the sensor resistances to a voltage suitable for readout with the microprocessors inputs, a simple voltage divider circuit with a 10 MOhm counter resistor was used. The data obtained from the sensors by the microprocessor was transmitted via a digital link to a radio chip located on the same foil. Communication to the outside world was performed by a dedicated radio chip operating at $13.56 \mathrm{MHz}$ frequency and compliant with ISO 18000-3 standard. This compatibly allows for readout with commercial RFID readers and smartphones equipped with a near field communication (NFC) receiver. To interface the sensor to the tag, a 14 pin's zero insertion force connector typically used for flat ribbon cables was used. In this way sensor foils can be modularly mounted onto the smart labels for testing.

Resistive sensors were made from interdigitated electrodes in contact with an inkjet printable sensing film. Photographs of the foils are given in figure $1 b$ and $c$. Figure $1 b$, depicts gold interdigitated electrodes on PEN. The electrodes were realized by sputtering a 150 $\mathrm{nm}$ layer of gold and patterned via photolithographic methods. Electrodes with a gap of 2.5 and $5 \mu \mathrm{m}$ were obtained. To overcome the use of costly fabrication methods and materials such as photolithography and gold, electrodes were also fabricated by screen printing of a silver micron paste. In combination with some post processing methods, reliable interdigitated electrodes with gaps down to 40 $\mu \mathrm{m}$ could be realized.

\section{Resistive amine sensors}

A resistive sensor capable of detecting the presence of amines was developed based on a dual layer sensor approach. First a thin layer of the p-type semiconducting polymer polytriarylamine (PTAA) was deposited. PTAA is well known for its good chemical and air stability[6]. The semiconductor exhibits no evident conductivity in the intrinsic state. A method for obtaining conduction is by chemical doping the semiconductor. This is typically performed before deposition (e.g. PANI, PEDOT:PSS)[7]. Here we investigated doping the semiconducting film by bringing it into contact with acidic compounds or films. A thin Nafion film was spin coated or on top of the PTAA film. The sulfonate groups of the Nafion at the interface of the PTAA film are suspected to dope the PTAA/nafion interface resulting in a conductive film. From transistor measurements a dopant density at the interface of the bilayer of $3 \times 10^{12}$ per $\mathrm{cm}^{2}$ was estimated.

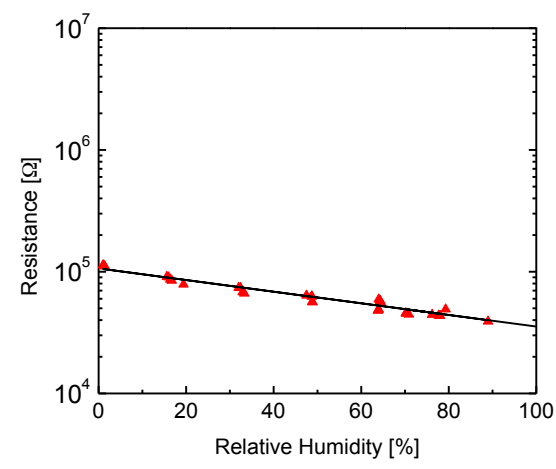

Figure 2. Resistance of the sensor bilayer biased at $0.5 \mathrm{~V}$ as a function of relative humidity. A variation in the order of a factor 3 is observed between 1 and $90 \%$ relative humidity. 
The role of the humidity on the conductivity of the film was investigated. The results are presented in figure 2. The bilayer shows proper conductivity under low as well as high relative humidity. Relatively small changes in resistances are obtained with varying humidity. The response of the bilayer was investigated for possible interference of alcohols and other organic volatile compounds. Again only minor changes were observed presumably due to swelling effects. When the films are however exposed to traces of bases such as ammonia or trimetylamine (TMA), large changes in resistance are observed. This is demonstrated in figure 3 .

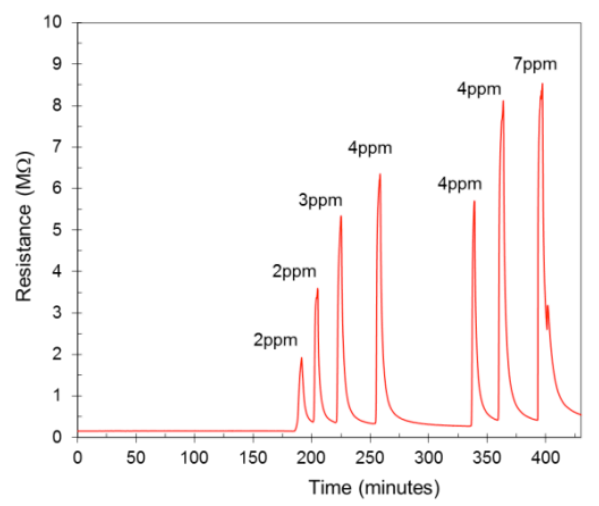

Figure 3: Bilayer sensor's response to low concentration of ammonia under 90\% Relative Humidity. Upon exposure to a few ppm of ammonia, a large increase in resistance is obtained. Recovery is obtained by flushing with (wet) air.

A tentative explanation of the large response towards ammonia or basic compounds is that the ammonia complexes with the sulfonate groups and as a consequence de-dopes the semiconductor film. The resulting large change in resistance enables simple readout. For the goal; the quantification of the freshness of fish, the marker of interest is TMA. The sensors response to TMA is given in figure 4 . A concentration of $1 \mathrm{ppm}$ in air with $90 \%$ relative humidity is used to mimic the environmental conditions of a food package and passed over the sensor. A clear response is observed. When the TMA is flushed from the system a slow recovery (washout effect) is obtained. The slow recovery is problematic for real time monitoring of TMA concentrations. In the case of food packaging where the concentration will only gradually increase, the slow recovery upon flushing is of lesser importance. The clear response to even as low concentrations as 1 ppm suggest it may be well suited for detecting the presence of TMA in the headspace of food packaging.

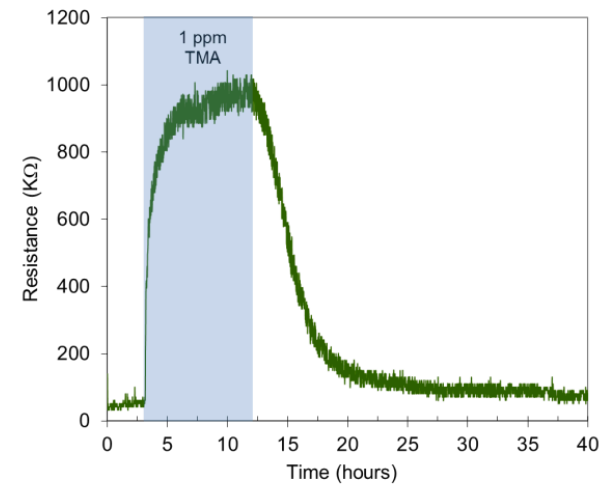

Figure 4: Bilayer sensor response to $1 \mathrm{ppm}$ of TMA. The TMA was mixed with wet air such that the relative humidity was approx. $90 \%$ to mimic condition typical in fish packaging. The sensor was readout with a simple MSP430 microprocessor.

\section{Sensor's response to cod fish}

The sensors response to aging fish was verified with cod fish filet. Cod fish filets pieces and a sensor were placed in closed jars in a fridge at $5{ }^{\circ} \mathrm{C}$ and the changes in resistance over time were analyzed. The sensors were readout every two minutes and the results are plotted in figure 5 . Two cases were compared. In the first case, fresh cod fish filet with the expiry label indicating expiry in two days was monitored. A low resistance was measured for over 30 hours. After 1.5 days a clear increase in resistance was measured suggesting the formation of TMA in the headspace. The experiment was also performed with cod fish 3 days beyond the expiry date. The observed change in resistance was substantially higher and faster suggesting a noticeable presence of TMA.

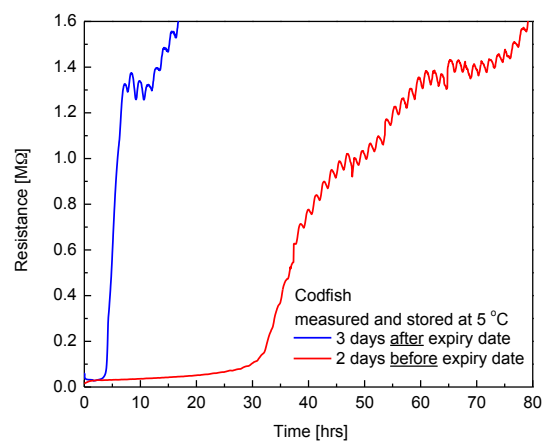

Figure 5: Bilayer sensor response to fresh and expired cod fish filet pieces. A clear difference in response is observed between the two samples. The sensor was readout with a simple MSP430 microprocessor. 
Form experiments with cod fish, it became evident that the concentration of TMA in the headspace and the associated freshness of fish, is dependent on a multitude of parameters such as the type of and quantity of fish. In addition temperature and humidity during transport and storage strongly affect the final outcome. Therefore the reliability of a freshness prediction can be greatly improved by monitoring additional indirect parameters such as the temperature and humidity on the tag.

\section{Resistive humidity sensors}

The freshness of products can be indirectly quantified by monitoring the temperature and humidity of a product during transport and storage. By using predictive models the freshness of fish can be estimated[7]. For temperature, the internal thermometer of the microprocessor is used. To measure the humidity in a cost effective manner, resistive sensors were fabricated. Humidity sensors were realizing by measuring the ionic conductivity of an electrolyte[8]. As an electrolyte we used Nafion. Without the PTAA film the Nafion film exhibits large changes in resistivity upon changes in humidity. The film showed good environmental stability under normal conditions. The sensors response to humidity is given in figure 6 . An exponential drop in conductivity is observed with decreasing humidity. We note that poisoning of the sensor in a basic (TMA) environment was not investigated.

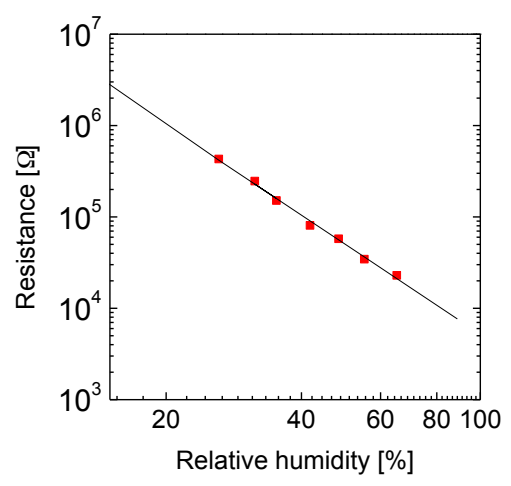

Figure 6: A Nafion humidity sensor's response in resistance as a function of relative humidity. The sensor was made from screen printed silver electrodes and readout with an MSP430 microprocessor. Large changes in resistance are observed with varying humidity.

\section{Conclusion}

In conclusion, we have demonstrated a radio frequency based smart label. The smart label consists of a printed antenna and circuitry. Logic and radio IC's were integrated together with a power source and sensors. Low power resistive sensors were developed to measure, humidity and the presence of volatile amine compounds. As a case study the smart labels are used to quantify the freshness of cod fish. In the future further integration and miniaturization of the tags and sensors is anticipated.

\section{References}

[1] D. Delen, B. C. Hardgrave, R.Sharda, RFID for Better Supply-Chain Management through Enhanced Information Visibility, Production and Operations Management 16 (2007) 613-624

[2] K. L. Yam, P. T. Takhistov, J. Miltz, Intelligent Packaging: Concepts and Applications, Journal of Food Science 70 (2005) 1-10

[3] G. Olafsdóttir, E. Martinsdóttira, J. Oehlenschlägerb, P. Dalgaardc, B. Jensenc, I. Undelandd, I.M. Mackiee, G. Henehanf, J. Nielsenc, H. Nilseng, Method to Evaluate Fish Freshness in Research and Industry Trends Food Sci. Tech. 8 (1997) 258-265

[4] A. Pacquit, J. Frisby, D. Diamond, K. T. Lau, A. Farrell, B. Quilty, D. Diamond, Development of a smart packaging for the monitoring of fish spoilage, Food Chemistry, 102 (2007) 466-470

[5] J. van den Brand, R. Kusters, M. Heeren, B. van Remoortere, A. Dietzel, Flip chip bonding of ultrathin Si dies onto PEN/PET substrates with low cost circuitry, Electronic SystemIntegration Technology Conference, 3 (2010) 1

[6] J. Veres, S.D. Ogier, S.W. Leeming, D.C. Cupertino, S. Mohialdin Khaffaf, Low-k Insulators as the Choice of Dielectrics in Organic Field-Effect Transistors, Advanced Functional materials (2003) 199-204

[7] Y. Cao, A. Andreattat, A. J. Heegert, P. Smith, Influence of chemical polymerization conditions on the properties of polyaniline, Polymer 30 (1989) 2305-2311

[7] P.S. Taoukis, K. Koutsoumanis, G.J.E. Nychas, Use of time-temperature integrators and predictive modelling for shelf life control of chilled fish under dynamic storage conditions, International Journal of Food Microbiology 53 (1999) 21-31

[8] M. Hijikagawa, S. Miyoshi, T. Sugihara, A. Jinda, A thin-film resistance humidity sensor, Sensors and Actuators 4, (1983) 307-315 